\title{
A New Sulfated $\alpha$-Ionone Glycoside from Sonchus erzincanicus Matthews
}

\author{
Ufuk Ozgen ${ }^{1}{ }^{1}$, Handan Sevindik ${ }^{1}$, Cavit Kazaz ${ }^{2}$, Demet Yigit ${ }^{3}$, Ali Kandemir ${ }^{3}$, Hasan Secen ${ }^{2}$ \\ and Ihsan Calis ${ }^{4, \dagger}$
}

1 Department of Pharmacognosy, Faculty of Pharmacy, Atatürk University, 25240 Erzurum, Turkey;

E-Mail: sevindikhandan@yahoo.com (H.S.)

2 Department of Chemistry, Faculty of Sciences, Atatürk University, 25240 Erzurum, Turkey;

E-Mails: ckazaz@atauni.edu.tr (C.K.); hsecen@atauni.edu.tr (H.S.)

3 Department of Biology, Faculty of Education, Erzincan University, 24030 Erzincan, Turkey;

E-Mails: dyigit@hotmail.com (D.Y.); kandemir_a@hotmail.com (A.K.)

4 Department of Pharmacognosy, Faculty of Pharmacy, Hacettepe University, 06100 Sihhıye, Ankara, Turkey; E-Mails: icalis@hacettepe.edu.tr (I.C.)

+ Present address: Department of Pharmacognosy and Pharmaceutical Botany, Faculty of Pharmacy, Near East University, Nicosia, North Cyprus, Turkey.

* Author to whom correspondence should be addressed; E-Mail: uozgen@atauni.edu.tr.

Received: 13 March 2010 / Accepted: 22 March 2010 / Published: 12 April 2010

\begin{abstract}
Sonchus erzincanicus (Asteraceae) is an endemic species in Turkey, where six Sonchus species grow. In this study, a phytochemical study was performed on the aerial parts of the plant. The study describes the isolation and structure elucidation of five flavonoids and two $\alpha$-ionone glycosides from $S$. erzincanicus. The compounds were isolated using several and repeated chromatographic techniques from ethyl acetate and aqueous phases that were partitioned from a methanol extract obtained from the plant. 5,7,3',4'-Tetrahydroxy-3-methoxyflavone (1) and quercetin 3-O- $\beta$-D-glucoside (2) were isolated from the ethyl acetate phase, while corchoionoside C 6'-O-sulfate (3), corchoionoside C (4), luteolin 7-O-glucuronide (5) and luteolin $7-O-\beta$-Dglucoside (6), apigenin 7-O-glucuronide (7) were isolated from the aqueous phase. Corchoionoside C 6'-O-sulfate (3), isolated for the first time from a natural source, was a new compound. The structures of the compounds were elucidated by means of ${ }^{1} \mathrm{H}-\mathrm{NMR},{ }^{13} \mathrm{C}-\mathrm{NMR}, 2 \mathrm{D}-\mathrm{NMR}$ (COSY, HMQC, HMBC) and ESI-MS.
\end{abstract}


Keywords: asteraceae; flavonoids; $\alpha$-ionone glycoside; Sonchus erzincanicus

\section{Introduction}

The Asteraceae family or Compositae is represented by about 900 genera and 13,000 species [1]. The genus Sonchus (Asteraceae) comprises 50 known species worldwide [1], and is represented by six species in the flora of Turkey, one of which, S. erzincanicus, is endemic, [2]. Sonchus species are variously known as "sütlük", "kuzu gevreği", and "eşek marulu" in Turkey [3]. It has been found that some Sonchus species contain sesquiterpene lactone glucosides, flavonoids, triterpenes and steroids [4,5]. No phytochemical study has so far been carried out on S. erzincanicus. This study describes the isolation and structure elucidation of five flavonoids and two $\alpha$-ionone glycosides, one being a new compound, from S. erzincanicus.

\section{Results and Discussion}

In our phytochemical studies on the aerial parts of Sonchus erzincanicus, we isolated flavonoids and $\alpha$-ionone glycosides by using several chromatographic methods. The flavonoids were identified as 5,7,3',4'-tetrahydroxy-3-methoxyflavone (1) [6], quercetin 3-O- $\beta$-D-glucoside (2) [7,8], luteolin 7-Oglucuronide (5) [9], luteolin 7-O- $\beta$-glucoside (6) [10] and apigenin 7-O-glucuronide (7) [11]. Compound 4 was identified as a known $\alpha$-ionone glycoside, corchoionoside C (4) [12]. Compound 3, corchoionoside C 6'-O-sulfate, was identified as a new natural compound (Figure 1).

Figure 1. Isolated compounds from Sonchus erzincanicus.<smiles>COc1c(-c2ccc(O)c(O)c2)oc2cc(O)cc(O)c2c1=O</smiles>

(1) 5,7, 3',4'-Tetrahydroxy-3-methoxy-flavone

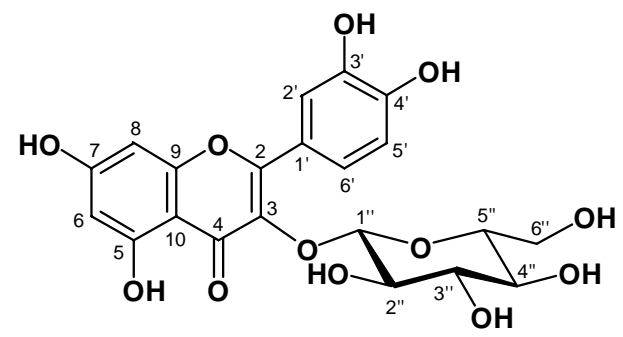

(2) Quercetin 3-O- $\beta$-D-glucoside

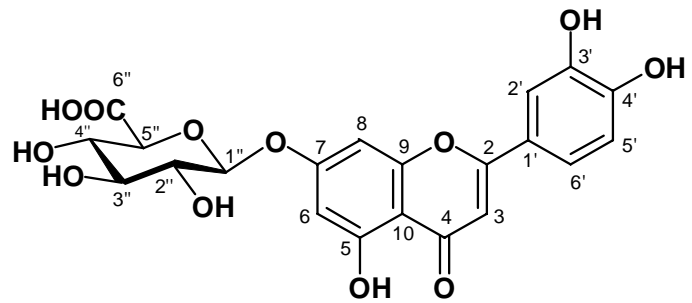

(5) Luteolin 7-O-glucuronide

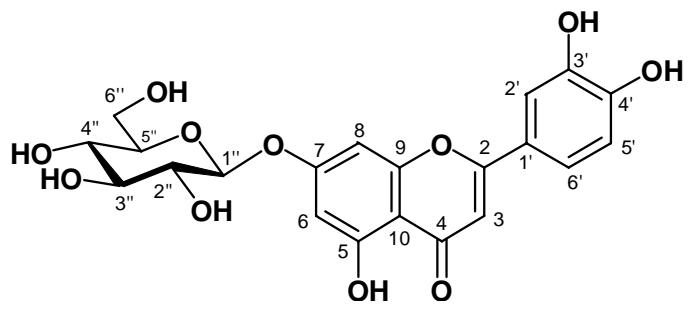

(6) Luteolin 7-O- $\beta$-D-glucoside 
Figure 1. Cont.

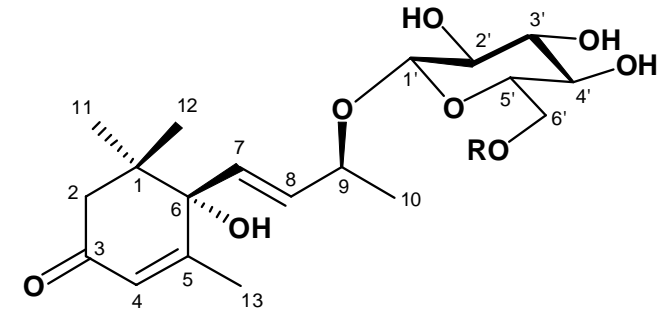

(3) $\mathrm{R}=\mathrm{SO}_{3} \mathrm{H}$ Corchoionoside $\mathrm{C}$ 6'-O-sulfate (4) $\mathrm{R}=\mathrm{H} \quad$ Corchoionoside

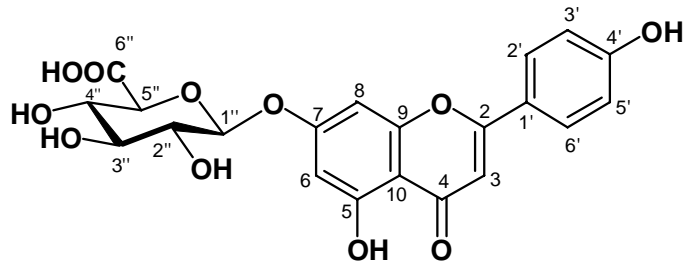

(7) Apigenin 7-O-glucuronide

The NMR data of compound 3 revealed the presence of a structure similar to that of compound 4 . HRMS spectra of protonated $3\left(\mathrm{MH}^{+}\right)$was 467.1564, which was in agreement with the calculated value: 467.1582. The ESI-MS of compound 3 showed the deprotonated molecule ion peak at $\mathrm{m} / \mathrm{z} 465$ $[\mathrm{M}-\mathrm{H}]^{-}$and a deprotonated positive ion peak with two added sodium atoms $[\mathrm{M}-\mathrm{H}+2 \mathrm{Na}]^{+}$at 511 . The assignments of all proton and carbon resonances (Table 1) were based on 2D NMR (COSY, HETCOR, HMBC) experiments. The anomeric proton signal at $\delta 4.26(\mathrm{~d}, J=7.7 \mathrm{~Hz})$ together with other resonances assigned to the sugar unit having a $\beta$-glucose moiety. Remaining signals were attributed to the ionone skeleton. All ${ }^{1} \mathrm{H}-\mathrm{NMR}$ and ${ }^{13} \mathrm{C}$-NMR signals were in agreement with the data given for the structure of corchoionoside $\mathrm{C}$ (4) except for C-6' and $\mathrm{H}_{2}-6$ ' due to esterification at this location [13]. While C-6' of corchoionoside C (4) resonates at $\delta 61.6 \mathrm{ppm}$, the same carbon of its sulfate derivative 3 resonates at $\delta 67.1 \mathrm{ppm}$ due to inductive effect of sulfate ester group. Four diastereomeric reseosides, diastereomers of corchoinoside $\mathrm{C}$, were recently synthesized by Yajima et al.: (6S,9S), corchoinoside C; (6S,9R); (6R,9S), (6R,9R). Comparing NMR spectral data of $\mathbf{3}$ and $\mathbf{4}$ with the ones of the four reseosides provided clear evidence that both corchoinoside $\mathrm{C} 4$ and corchoionoside $\mathrm{C}$ 6'-O-sulfate 3 are in agreement with the structure of $(6 \mathrm{~S}, 9 \mathrm{~S})$ reseoside [14]. Thus, the structure of $\mathbf{3}$ was established as corchoionoside C 6'-O-sulfate. It is the first time that this compound has been isolated from Nature.

Table 1. NMR Spectroscopic data for compound $3\left({ }^{1} \mathrm{H}-\mathrm{NMR}: 400 \mathrm{MHz},{ }^{13} \mathrm{C}-\mathrm{NMR}\right.$ : $\left.100 \mathrm{MHz}\right)$.

\begin{tabular}{llll}
\hline $\mathbf{C} / \mathbf{H}$ atom & $\boldsymbol{\delta} \mathbf{C}$ & $\boldsymbol{\delta H} \mathbf{~ p p m}, \mathbf{J}(\mathbf{H z})$ & $\mathbf{H M B C}(\mathbf{H} \rightarrow \mathbf{C})$ \\
\hline 1 & 41.3 & & \\
2 & 49.6 & $2.63 \mathrm{~d}(16.7)$ & $\mathrm{C}-11, \mathrm{C}-12$ \\
& & $2.16 \mathrm{~d}(16.7)$ & \\
3 & 200.2 & & \\
4 & 126.0 & $5.87 \mathrm{bs}$ & $\mathrm{C}-6, \mathrm{C}-2$ \\
5 & 165.9 & & \\
6 & 78.8 & & \\
7 & 132.5 & $5.98 \mathrm{~d}(15.6)$ & $\mathrm{C}-9, \mathrm{C}-5$ \\
8 & 132.4 & $5.70 \mathrm{dd}(15.6,7.2)$ & $\mathrm{C}-6, \mathrm{C}-10$ \\
9 & 73.5 & 4.50 quintet $(6.8)$ & $\mathrm{C}-1$ ', C-7 \\
10 & 21.0 & $1.28 \mathrm{~d}(6.2)$ & $\mathrm{C}-8$ \\
11 & 22.3 & $1.03 \mathrm{~s}$ & $\mathrm{C}-2, \mathrm{C}-6$ \\
12 & 23.6 & $1.01 \mathrm{~s}$ & $\mathrm{C}-2, \mathrm{C}-6$ \\
13 & 18.4 & $1.94 \mathrm{~d}(1.1)$ & $\mathrm{C}-6, \mathrm{C}-4$ \\
\hline
\end{tabular}


Table 1. Cont.

\begin{tabular}{llll}
\hline $\mathrm{C} / \mathrm{H}$ atom & $\delta \mathrm{C}$ & $\delta \mathrm{H} \mathrm{ppm}, \mathrm{J}(\mathrm{Hz})$ & $\mathrm{HMBC}(\mathrm{H} \rightarrow \mathrm{C})$ \\
\hline Glucose & & & \\
$1^{\prime}$ & 100.1 & $4.26 \mathrm{~d}(7.7)$ & \\
$2^{\prime}$ & 73.7 & & \\
$3^{\prime}$ & 74.9 & $3.28-3.36^{\mathrm{a}}$ & \\
$4^{\prime}$ & 70.3 & \\
$5^{\prime}$ & 76.9 & $4.29 \mathrm{dd},(10.9,1.8)$ \\
$6^{\prime}$ & 67.1 & $4.09 \mathrm{dd}(10.9,5.5)$ \\
\hline
\end{tabular}

${ }^{\text {a }}$ Signal patterns are not clear due to overlapping.

\section{Experimental}

\subsection{General}

${ }^{1} \mathrm{H}-\mathrm{NMR}$ and ${ }^{13} \mathrm{C}$-NMR spectra were recorded with a Varian Mercury plus spectrometer at 400 and at $100 \mathrm{MHz}$, respectively. Mass spectra were recorded with Micromass ZQ Mass Spectrometer (Manchester, UK). Sephadex LH-20 (Sigma-Aldrich) and Silica gel (Kiesel gel 60, 0.063-0.2 mm Merck 7734 and 0.040-0.063 mm Merck 9385 and LiChroprep RP-18, 25-40 $\mu \mathrm{m}$, Merck 9303) were used for column chromatography, while silica gel $60 \mathrm{~F}_{254}$ (Merck, 5554) was used for TLC. TLC spots were detected with a UV lamp, spraying $1 \%$ Vanillin $/ \mathrm{H}_{2} \mathrm{SO}_{4}$ and heated at $120{ }^{\circ} \mathrm{C}$ for $1-2 \mathrm{~min}$.

\subsection{Plant material}

The aerial parts of S. erzincanicus were collected from Ekşisu (Erzincan Province, Turkey) in 2006 and was identified by Dr. A. Kandemir. A voucher specimen was deposited in the Herbarium of Erzincan University, Faculty of Education (EEFH 7794).

\subsection{Extraction and isolation}

Dried aerial parts $(260 \mathrm{~g})$ of the plant material were extracted by refluxing with methanol (2 L x 3 ) on a mantle. The methanol extract was concentrated and dried under reduced pressure to give a residue (44.3 g). Methanol extract (44.0 g) was dissolved in $\mathrm{H}_{2} \mathrm{O}-\mathrm{MeOH}(9: 1)$ and partitioned with chloroform and then ethyl acetate, which were separately concentrated and dried under reduced pressure to give $9.4 \mathrm{~g}$ and $0.9 \mathrm{~g}$ residues, respectively. The remaining aqueous phase was $32 \mathrm{~g}$. There were too few compounds to isolate and identify in chloroform phase.

The ethyl acetate phase $(0.9 \mathrm{~g})$ was subjected to silica gel column chromatography using $\mathrm{CHCl}_{3}$ $\mathrm{MeOH}-\mathrm{H}_{2} \mathrm{O}$ (80:20:2, 70:30:3, 50:50:5) solvent systems. Fifty nine fractions were collected. Fraction 6 (35.7 mg) gave compound $\mathbf{1}(9 \mathrm{mg})$ while fractions 18-24 (27 mg) gave compound $2(15 \mathrm{mg})$.

The remaining aqueous phase $(32 \mathrm{~g})$ was subjected to reversed phase silica gel column chromatography using $0-100 \%$ aqueous $\mathrm{MeOH}$ as solvent systems. Fractions were monitored by TLC on silica gel plates and similar fractions were combined to give fraction A (Fr. 18-28, $5.5 \mathrm{~g}$ ), fraction $\mathbf{B}$ (Fr. 30-40, $334 \mathrm{mg}$ ) and fraction C (Fr. 45-52, $270 \mathrm{mg}$ ). 
Fraction A was subjected to silica gel column chromatography with $\mathrm{CHCl}_{3}: \mathrm{MeOH}: \mathrm{H}_{2} \mathrm{O}(70: 30: 3$, 65:35:5) solvent system. Fr. 36-47 gave compound 3 (18 mg).

Fraction B was subjected to a gel chromatography (Sephadex LH-20) eluting with $\mathrm{MeOH}$ and 15 fractions were collected. The fractions 2-5 (B1, $157 \mathrm{mg}$ ) were further purified by successive column chromatography on silica gel and Sephadex LH-20, respectively, yielding pure 4 (18 $\mathrm{mg}$ ). The fractions 8-10 (B2, $25 \mathrm{mg})$ gave compound 5.

Fraction $\mathrm{C}$ was subjected to a silica gel column chromatography with $\mathrm{CHCl}_{3}-\mathrm{MeOH}-\mathrm{H}_{2} \mathrm{O}(70: 30: 3)$ and 60 fractions were collected. The fractions 10-14 (C1, $50 \mathrm{mg})$ were subjected to gel chromatography (Sephadex LH-20) with $\mathrm{MeOH}$ to give compound 6 (14 mg). The fractions 41-50 (C2, $41 \mathrm{mg}$ ) were subjected to a gel chromatography (Sephadex LH-20) with $\mathrm{MeOH}$ to give compound 7 (10 mg).

Compound 1: Yellow powder; ${ }^{1} \mathrm{H}-\mathrm{NMR}\left(\mathrm{CD}_{3} \mathrm{OD}\right): \delta 7.60(1 \mathrm{H}, \mathrm{bs}, \mathrm{H}-2$ '), $7.52(1 \mathrm{H}, \mathrm{d}, \mathrm{H}-6$ ', $J=8.4 \mathrm{~Hz}), 6.89\left(1 \mathrm{H}, \mathrm{d}, \mathrm{H}-5{ }^{\prime}, J=8.4 \mathrm{~Hz}\right), 6.37(1 \mathrm{H}, \mathrm{bs}, \mathrm{H}-8), 6.18(1 \mathrm{H}, \mathrm{bs}, \mathrm{H}-6), 3.77\left(\mathrm{~s}, \mathrm{OCH}_{3}\right)$; ${ }^{13} \mathrm{C}-\mathrm{NMR}\left(\mathrm{CD}_{3} \mathrm{OD}\right): \delta 178.8$ (C-4), 164.9 (C-7), 161.8 (C-5), 157.2 (C-9), 156.8 (C-2), 148.7 (C-4'), 145.3 (C-3'), 138.3 (C-3), 121.7 (C-1'), 121.1 (C-6'), 115.3 (C-5'), 115.2 (C-2'), 104.6 (C-10), 98.6 (C6), $93.6(\mathrm{C}-8), 59.3\left(\mathrm{OCH}_{3}\right) .{ }^{1} \mathrm{H}-\mathrm{NMR}$ and ${ }^{13} \mathrm{C}-\mathrm{NMR}$ agree with data given in the literature for

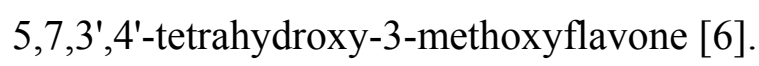

Compound 2: Yellow powder; ${ }^{1} \mathrm{H}-\mathrm{NMR}\left(\mathrm{CD}_{3} \mathrm{OD}\right): \delta 7.70\left(1 \mathrm{H}, \mathrm{d}, \mathrm{H}-2^{\prime}, J=1.9 \mathrm{~Hz}\right), 7.58(1 \mathrm{H}, \mathrm{dd}, \mathrm{H}-$ $\left.6^{\prime}, J=8.5 \mathrm{~Hz}, 1.9 \mathrm{~Hz}\right), 6.86(1 \mathrm{H}, \mathrm{d}, \mathrm{H}-5 ', J=8.5 \mathrm{~Hz}), 6.36(1 \mathrm{H}, \mathrm{d}, \mathrm{H}-8, J=2.2 \mathrm{~Hz}), 6.17$ (1H, d, H-6, $J=2.2 \mathrm{~Hz}), 5.22(1 \mathrm{H}, \mathrm{d}, \mathrm{H}-1 ", J=7.3 \mathrm{~Hz}), 3.85-3.30\left(6 \mathrm{H}\right.$, sugar protons); ${ }^{13} \mathrm{C}-\mathrm{NMR}\left(\mathrm{CD}_{3} \mathrm{OD}\right): \delta$ 178.1 (C-4), 166.2 (C-7), 161.8 (C-5), 157.6 (C-2), 157.4 (C-9), 148.7 (C-4'), 144.7 (C-3'), 134.4 (C3), 122.0 (C-1'), 121.9 (C-6'), 116.3 (C-5'), 114.8 (C-2'), 104.1 (C-10), 103.2 (C-1"), 99.2 (C-6), 93.8 (C-8), 77.2 (C-5"), 76.9 (C-3"), 74.5 (C-2"), 70.0 (C-4"), 61.4 (C-6"). ${ }^{1} \mathrm{H}-\mathrm{NMR}$ and ${ }^{13} \mathrm{C}-\mathrm{NMR}$ agree with data given in the literature for quercetin $3-O-\beta$-D-glucoside $[7,8]$.

Compound 3: Amorphous colourless solid; $[\alpha]_{\mathrm{D}}{ }^{22}=+38(\mathrm{c}=1, \mathrm{MeOH})$, ESI-MS $\left(\mathrm{C}_{19} \mathrm{H}_{30} \mathrm{O}_{11} \mathrm{~S}\right), \mathrm{m} / \mathrm{e}$ : $465[\mathrm{M}-\mathrm{H}]^{-}$and $511[\mathrm{M}-\mathrm{H}+2 \mathrm{Na}]^{+}$. HRMS: calculated for $\mathrm{C}_{19} \mathrm{H}_{31} \mathrm{O}_{11} \mathrm{~S}^{+}$: 467.1582; found: 467.1564 . For ${ }^{1} \mathrm{H}-\mathrm{NMR}\left(\mathrm{CD}_{3} \mathrm{OD}\right)$ and ${ }^{13} \mathrm{C}-\mathrm{NMR}\left(\mathrm{CD}_{3} \mathrm{OD}\right)$. See (Table 1$)$.

Compound 4: Amorphous colourless solid, ESI-MS $\left(\mathrm{C}_{19} \mathrm{H}_{30} \mathrm{O}_{8}\right), \mathrm{m} / \mathrm{e}: 409[\mathrm{M}+\mathrm{Na}]^{+}$and $385[\mathrm{M}-\mathrm{H}]^{-}$, ${ }^{1} \mathrm{H}-\mathrm{NMR}\left(\mathrm{CD}_{3} \mathrm{OD}\right): \delta 5.97(1 \mathrm{H}, \mathrm{d}, \mathrm{H}-7, J=15.2 \mathrm{~Hz}), 5.86(1 \mathrm{H}, \mathrm{s}, \mathrm{H}-4), 5.72(1 \mathrm{H}, \mathrm{dd}, \mathrm{H}-8$, $J=15.5 \mathrm{~Hz}, J=7.0 \mathrm{~Hz}), 4.53(1 \mathrm{H}$, quintet, $\mathrm{H}-9, J=6.6 \mathrm{~Hz}), 4.26\left(1 \mathrm{H}, \mathrm{d}, \mathrm{H}-1^{\prime}, J=7.7 \mathrm{~Hz}\right), 3.85(1 \mathrm{H}$, dd, H- $\left.6_{\mathrm{a}}{ }^{\prime}, J=11.9 \mathrm{~Hz}, J=2.2 \mathrm{~Hz}\right), 3.62\left(1 \mathrm{H}, \mathrm{dd}, \mathrm{H}-6_{\mathrm{b}}{ }^{\prime}, J=11.9 \mathrm{~Hz}, J=6.0 \mathrm{~Hz}\right), 3.25-3.34$ (sugar protons, overlapped, 4H, H-2', H-3', H-4', H-5'), 2.61 (1H, d, H-2a, J = 17.6 Hz), 2.16 (1H, d, H-2b, J $=17.6 \mathrm{~Hz}), 1.94(3 \mathrm{H}, \mathrm{bs}, \mathrm{H}-13), 1.02(3 \mathrm{H}, \mathrm{d}, \mathrm{H}-10, J=8.4 \mathrm{~Hz}), 0.91(3 \mathrm{H}, \mathrm{s}, \mathrm{H}-11), 0.88(3 \mathrm{H}, \mathrm{s}, \mathrm{H}-$ 12); ${ }^{13} \mathrm{C}-\mathrm{NMR}\left(\mathrm{CD}_{3} \mathrm{OD}\right): \delta 200.0$ (C-3), 166.0 (C-5), 132.6 (C-7), 132.5 (C-8), 125.9 (C-4), 100.1 (C1'), 77.1 (C-6), 77.0(C-5'), 73.8 (C-3'), 73.4 (C-2'), 70.7 (C-9), 70.5 (C-4'), 61.6 (C-6'), 49.8 (C-2), 41.2 (C-1), 23.5 (C-12), 22.5 (C-11), 22.3 (C-10), 18.4 (C-13). ${ }^{1} \mathrm{H}-\mathrm{NMR}$ and ${ }^{13} \mathrm{C}-\mathrm{NMR}$ agree with data given in the literature for corchoionoside $\mathrm{C}$ [12]. 
Compound 5: Yellow powder; ${ }^{1} \mathrm{H}-\mathrm{NMR}\left(\mathrm{DMSO}-d_{6}\right): \delta 7.40\left(1 \mathrm{H}, \mathrm{d}, \mathrm{H}-2^{\prime}, J=2.0 \mathrm{~Hz}\right), 7.36(1 \mathrm{H}, \mathrm{dd}, \mathrm{H}-$ $\left.6^{\prime}, J=8.4 \mathrm{~Hz}, J=2.0 \mathrm{~Hz}\right), 6.84(1 \mathrm{H}, \mathrm{d}, \mathrm{H}-5$ ',$J=8.4 \mathrm{~Hz}), 6.74(1 \mathrm{H}, \mathrm{d}, \mathrm{H}-8, J=1.9 \mathrm{~Hz}), 6.69(1 \mathrm{H}, \mathrm{s}$, H-3), 6.39 (1H, d, H-6, $J=1.9 \mathrm{~Hz}), 5.06$ (1H, d, H-1", $J=7.3 \mathrm{~Hz}), 3.60$ (1H, d, H-5", $J=9.9 \mathrm{~Hz})$, 3.39-3.14 (m, $3 \mathrm{H}$, sugar protons, overlapped with DMSO- $d_{6}$ signals); ${ }^{13} \mathrm{C}-\mathrm{NMR}$ (DMSO- $\left.d_{6}\right): \delta 182.5$ (C-4), 172.5 (C-6"), 165.1 (C-2), 163.6 (C-7), 161.7 (C-5), 157.6 (C-9), 150.8 (C-4'), 146.6 (C-3'), 121.7 (C-1'), 119.7 (C-6'), 116.7 (C-5'), 114.1 (C-2'), 105.9 (C-10), 103.6 (C-3), 100.2 (C-1"), 100.2 (C-6), 95.2 (C-8), 77.1 (C-3"), 74.5 (C-5"), 73.6 (C-2"), 72.6 (C-4"). ${ }^{1} \mathrm{H}-\mathrm{NMR}$ and ${ }^{13} \mathrm{C}-\mathrm{NMR}$ agree with data given in the literature for luteolin 7-O-glucuronide [9].

Compound 6: Yellow powder; ${ }^{1} \mathrm{H}-\mathrm{NMR}\left(\mathrm{DMSO}-d_{6}\right): \delta 7.42(1 \mathrm{H}, \mathrm{bd}, \mathrm{H}-6$ ', $J=8.8 \mathrm{~Hz}), 7.40(1 \mathrm{H}$, bs, H-2'), $6.87\left(1 \mathrm{H}, \mathrm{d}, \mathrm{H}-5^{\prime}, J=8.4 \mathrm{~Hz}\right), 6.77(1 \mathrm{H}, \mathrm{d}, \mathrm{H}-8, J=1.8 \mathrm{~Hz}), 6.73(1 \mathrm{H}, \mathrm{s}, \mathrm{H}-3), 6.42(1 \mathrm{H}, \mathrm{d}$, $\mathrm{H}-6, J=1.8 \mathrm{~Hz}), 5.06(1 \mathrm{H}, \mathrm{d}, \mathrm{H}-1 ", \quad J=7.3 \mathrm{~Hz}), 3.70-3.15\left(6 \mathrm{H}\right.$, sugar protons); ${ }^{13} \mathrm{C}-\mathrm{NMR}$ (DMSO$\left.d_{6}\right): \delta 182.5(\mathrm{C}-4), 165.2(\mathrm{C}-2), 163.6(\mathrm{C}-7), 161.8(\mathrm{C}-5), 157.6(\mathrm{C}-9), 151.1\left(\mathrm{C}-4^{\prime}\right), 146.6\left(\mathrm{C}-3^{\prime}\right), 121.7$ (C-1'), 119.9 (C-6'), 116.6 (C-5'), 114.1 (C-2'), 106.0 (C-10), 103.7 (C-3), 100.6 (C-1"), 100.2 (C-6), 95.4 (C-8), 77.8 (C-5"), 77.1 (C-3"), 73.8 (C-2"), 70.2 (C-4"), 61.3 (C-6"). ${ }^{1} \mathrm{H}-\mathrm{NMR}$ and ${ }^{13} \mathrm{C}-\mathrm{NMR}$ agree with data given in the literature for luteolin $7-O-\beta$-D-glucoside [10].

Compound 7: Yellow powder; ${ }^{1} \mathrm{H}-\mathrm{NMR}$ (DMSO- $\left.d_{6}\right)$ : $\delta 7.86(2 \mathrm{H}$, quasi d, H-2'/6', $J=8.8 \mathrm{~Hz}), 6.88$ $(2 \mathrm{H}$, quasi d, H-3'/5', $J=8.8 \mathrm{~Hz}), 6.84(1 \mathrm{H}, \mathrm{d}, \mathrm{H}-8, J=2.0 \mathrm{~Hz}), 6.62(1 \mathrm{H}, \mathrm{s}, \mathrm{H}-3), 6.49$ (1H, d, H-6, $J$ $=2.0 \mathrm{~Hz}$ ), $5.10\left(1 \mathrm{H}, \mathrm{d}, \mathrm{H}-1 ", J=6.6 \mathrm{~Hz}\right.$ ), 3.90-3.25 (sugar protons, 4H, H-2", H-3", H-4", H-5"); ${ }^{13} \mathrm{C}-$ NMR (DMSO- $\left.d_{6}\right): \delta 183.0$ (C-4), 175.1 (C-6"), 165.9 (C-2), 163.7 (C-7), 161.8 (2C, C-4' and C-5), 157.8 (C-9), 128.5 (2C, C-2'/6'), 121.1 (C-1'), 116.4 (2C, C-3'/5'), 106.0 (C-10), 102.4 (C-3), 100.4 (C1"), 100.2 (C-6), 94.9 (C-8), 76.4 (C-5"), 75.4 (C-3"), 73.4 (C-2"), 72.3 (C-4"). ${ }^{1}$ H-NMR [11] and ${ }^{13} \mathrm{C}-$ NMR [15] agree with data given in the literature for apigenin 7-O-glucuronide.

\section{Acknowledgements}

The authors are grateful to Erhan Palaska (Hacettepe University, Faculty of Pharmacy, Ankara, Turkey) for ESI mass spectra. We thank Arata Yajima for providing graphical NMR spectra of the four reseosides by which we could establish the structures of $\mathbf{3}$ and $\mathbf{4}$.

\section{References}

1. Evans, W.C. Trease and Evans' Pharmacognosy, 13th ed.; Balliére Tindall: London, UK, 1989; pp. 226-227.

2. Matthews, V.A. Sonchus L. In Flora of Turkey and the East Aegean Islands; Davis, P.H., Ed.; University Press: Edinburgh, UK, 1975; Volume 5, pp. 690-696.

3. Akartürk, R. Şifalı Bitkiler, Flora ve Sağlı̆̆ımız; Orman Genel Müdürlüğü Mensupları Yardımlaşma Vakfı (in Turkish): Ankara, Turkey, 2001.

4. Helal, A.M.; Nakamura, N.; El-Askary, H.; Haatori, M. Sesquiterpene lactone glucosides from Sonchus asper. Phytochemistry 2000, 53, 473-477. 
5. Devkota, K.P. Cholinesterase inhibiting and antiplasmodial steroidal alkaloids from Sarcococca hookeriana. Ph.D. Thesis. Research Institute of Chemistry: University of Karachi, Karachi, Pakistan, 2005.

6. Zheng, Z.P.; Cheng, K.W.; Chao, J.; Wu, J.; Wang, M. Tyrosinase inhibitors from paper mulberry (Broussonetia papyrifera). Food Chem. 2008, 106, 529-535.

7. Caldwell, S.T.; Petersson, H.M.; Farrugia, L.J.; Mullen, W.; Croizer, A.; Hartley, R.C. Isotopic labelling of quercetin 3-glucoside. Tetrahedron 2006, 62, 7257-7265.

8. Ko, J.H.; Kim, B.G.; Kim, J.H.; Kim, H.J.; Lim, C.E.; Lim, J.; Lee, C.; Lim, Y.H.; Ahn, J.H. Four glucosyltransferases from rice: cDNA cloning, expression, and characterization. J. Plant Physiol. 2008, 165, 435-444.

9. Lu, Y.; Foo, L.Y. Flavonoid and phenolic glycosides from Salvia officinalis. Phytochemistry 2000, 55, 263-267.

10. Asada, H.; Miyase, T.; Fukushima, S. Sesquiterpene lactones from Ixeris tamagawaensis KITAM. Chem. Pharm. Bull. 1984, 32, 1724-1728.

11. Malikov, V.M.; Yuldashev, M.P. Phenolic compounds of plants of the Scutellaria L. genus. Distribution, structure, and properties. Chem. Nat. Comp. 2002, 38, 358-406.

12. Çalış, İ.; Kuruüzüm-Uz, A.; Lorenzetto, P.A.; Rüedi, P. (6S)-Hydroxy-3-oxo- $\alpha$-ionol glucosides from Capparis spinosa fruits. Phytochemistry 2002, 59, 451-457.

13. Yahara, S.; Ding, N.; Nohara, T.; Masuda, K.; Ageta, H. Taraxastane glycosides from Eclipta alba. Phytochemistry 1997, 44, 131-135.

14. Yajima A.; Oono Y.; Nakagawa R.; Nukada T.; Yabuta G. A simple synthesis of four stereoisomers of roseoside and their inhibitory activity on leukotriene release from mice bone marrow-derived cultured mast cells. Bioorg. Med. Chem. 2009, 17, 189-194.

15. Sohn, U.D.; Whang, W.K.; Ham, I.H.; Min, Y.S.; Bae, K.L.; Yim, S.H.; Lee, Y.P. Process for preparing apigenin-7-O-beta-D-glucuronide from Clerodendron trichotomum folium. WO Pat. 2003099306, 2003.

Sample Availability: Samples of the compounds are available from the authors.

(C) 2010 by the authors; licensee Molecular Diversity Preservation International, Basel, Switzerland. This article is an open-access article distributed under the terms and conditions of the Creative Commons Attribution license (http://creativecommons.org/licenses/by/3.0/). 